A. Lespagrol. Les glucides du lait de femme. Thèse Pharmacopat supérieur, Lille, 1930.

[i] Aunrbach et Bodlatnder. Zeitschr. f. ang. Chem., 1923, 602.

[8] M. Polonovski, A. Lespagnol et H. Warembourg, C. R. de la Sor, de Piol., 1931, 107, 303.

\title{
L'EFFET DU CHAUFFAGE PRÉLIMINAIRE SUR LA DISPERSION DU CASÉINATE DE CALCIUM DANS LE LAIT ÉCRÉMIÉ (1)
}

\author{
par \\ B. NICHOLS (2), E. D. BAILEY (2), G. E. HOLM (3), \\ G. R. GREENBANK (3) et E. F. DEYSHER (3).
}

La caséine est en suspension dans le lait sous forme de caséinate de calcium; à un degré élevé de dispersion; elle peut être séparée au moyen d'un ultra-filtre de porcelaine ou de collodion, ou parlacentrifugation à très grande vitesse (4).

Svedberg, Wiegner et BechHold ont essayé d'estimer le degré de dispersion du caséinate de calcium (5). D'études préliminaires d'ultra-centrifugation, faites en 1924, SvEDBERG et FAHRAEUS ont conclu que les particules étaient de l'ordre de grandeur d'un rayon de 10 à $70 \mathrm{~m} \mu\left(\mathrm{m} \mu=10^{-7} \mathrm{~cm}\right.$.). (6).

Wiegner est d'avis qu'en majorité les particules sont des amicrons et que leur dimension varie probablement de $5 \mathrm{~m} \mu$ à $100 \mathrm{~m} \mu$ en diamètre. Il constata que le nombre de particules était constant même lorsque les laits avaient été chauffés ou additionnés de petites quantités d'acide. BEO HOLD, se basant sur les données de l'ultrafiltration de séries de protéines et de colloïdes inorganiques, émet l'avis que les particules du caséinate de calcium ont un diamètre probablement plus grand que $40 \mathrm{~m} \mu$.

La dispersion du caséinate de calcium présente un intérêt considérable, tant au point de vue théorique que pratique. Un des pro-

(1) Nous faisons paraître in extenso le présent article publié antérieurement dans The Journal of Physical Chemistry, 35, 1303, 1931, et dont la traduction est due à M. R. N. Göransson, en raison de son grand intérêt. Jamais trop on ne fouillera assez la structure du lait et son comportement en présence des circonstances les plus varjées.

Nous remercions les auteurs de nous avoir envoyé les planches pour les reproduire en français et le Jaurnal of Physical Chemistry de nous permettre obligeamment de faire paraître ce travail en français. (Ch. P.)

(2) Experimental Station, E. I. du Pont de Nemours Company.

(3) Bureau of Dairy Indust:y, U.S. Department of Agriculture.

(4) Friedenthal : Ber., 44, 904, (1911) Van Slyke et Bosworth : New York (Geneva) Agr. Exp. Stat. Techn. Bull. 39 et J. of Biol. Chem. 20, 35, (1915).

(5) Svedberg : Kolloid-Z.,51, 10 (1930); Wiegner : Z. Nahr. Genussm, 27, 425 (1914); Bеснноц : Z. physik. Chem., 60, 257 (1907).

(6) $\mathrm{m} \mu=\mu \mu$, soit le millionième du millimètre. 
blèmes capitaux de l'industrie du lait évaporé, ainsi que d’autres branches de l'industrie laitière est le développement d'une stabilité convenable vis-à-vis de la chaleur.

On n'a que peu de connaissances concernant le mécanisme qui provoque la coagulation, quoique les méthodes industrielles mises en ouvre pour la prévenir soient bien établies. Dans la préparation du lait évaporé, le lait est chauffé à la température de $95^{\circ} \mathrm{C}$., préalablement à la concentration. Cette pratique a pour but de donner au produit achevé une plus grande stabilité, lors de la stérilisation terminale. Lorsqu'on a recours à des températures d'environ $70^{\circ} \mathrm{C}$., il paraît y avoir une tendance à la réduction de la stabilité (1).

Ces traitements par la chaleur paraissent également influencer certaines propriétés physiques de la dispersion. Par exemple, le chauffage du lait à $70^{\circ} \mathrm{C}$. diminue la viscosité du lait, tandis que le chauffage à des températures plus élevées l'accentue. Ces modifications de la viscosité sont accompagnées de modifications de la densité (données non encore publiées). Quoique la stabilisation soit influencée dans de très grandes proportions par les électrolytes présents dans le sérum, il est possible qu'elle dépende en partie de modifications de la dimension des particules. De ce fait, des études d'ultracentrifugation furent entreprises, en vue de déterminer la courbe de distribution du caséinate de calcium non modifié et de vérifier si le chauffage influence la distribution des particules dans le lait écrémé.

\section{EXPERIENCES.}

Préparation des échantillons. - Du lait écrémé frais fut chauffé à une température voisine de $40^{\circ} \mathrm{C}$. et passé trois fois par un clarificateur de Laval, en vue d'éliminer aussi complètement que possible la matière grasse finement-divisée, les leucocytes, et tout autre matériel à particules relativement volumineuses. Un échantillon fut chauffé dans un bain-marie à $65^{\circ} \mathrm{C}$, et un autre à $95^{\circ} \mathrm{C}$. dans des flacons pourvus de condensateurs à reflux, pendant dix minutes. Ces échantillons, ainsi que l'échantillon-témoin furent conservés dans la glace, jusqu'à l'utilisation pour les expériences. Puisque la quantité de calcium associée à la caséine est une fonction de la teneur en électrolytes du sérum, du sérum du lait originel fut utilisé comme liquide de dilution pour maintenir l'équilibre primitif au cours des expériences. Ce sérum fut obtenu par ultrafiltration sur un ultra-filtre de collodion, et conservé dans la glace jusqu'à l'utilisation. Lorsqu'un échantillon fut laissé à la température ordinaire pendant quelque temps, il s'y forma un précipité floconneux, selon toute présomption, de phosphate de calcium.

(1) Deysher, Webb et Holm : Journ. Dairy Sc, , 12, 80 (1929). 
Détermination par ultra-centrifugation. - Dans la détermination centrifuge de la distribution des particules d'après la dimension d'un matériel polydispersé, on utilisa, pour déterminer le rayon d'une particule, une forme modifiée de la loi de Stokes (1):

$$
r=\sqrt{\frac{9_{\gamma_{1}} \ln [(\mathrm{x}+\mathrm{a}) / \mathrm{a}]}{2\left(\rho_{\mathrm{r}}-\rho_{1}\right){ }_{(1)}{ }^{2} \mathrm{t}}}
$$

dans laquelle $r$ est le rayon de la particule en centimètres, $r$ la viscosité du milieu, $\rho_{p}$ la densité de la particule, $\rho_{1}$ la densité du milieu, $\omega\left(=2 \pi \frac{\mathrm{N}}{60}\right)$ la vitesse angulaire, $n$ la vitesse de la centrifuge en nombre de rotations par minute, (r. p. m.), $t$ la durée de la centrifugation en secondes, $x$ la distance, en centimètres, du ménisque le long de la cellule, et a la distance du ménisque au centre de rotation. Les autres données nécessaires en plus de celles obtenues avec l'ultra-centrifuge pour la détermination de la courbe de distribution sont les viscosités des solutions, la densité des particules de caséinate de calcium et la densité du sérum dans lequel les particules sont en dispersion. Ces données sont fournies au tableau I.

\section{TABLEAU I.}

\begin{tabular}{|c|c|c|}
\hline & Lait écrémé & Sérum \\
\hline Solides* & $9,40 \%$ & $6,22 \%$ \\
\hline Protéines & $3,39 \%$ & $0,40 \%$ \\
\hline Densité à $30^{\circ} \mathrm{C}$. & 1,0317 & 1,022 \\
\hline
\end{tabular}

Densité apparente du caséinate de calcium ............ 1,505

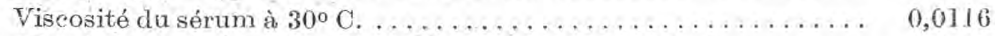

Viscosité de $4 \mathrm{p}$. de sérum pour 1 de lait écrémé à $30^{\circ} \mathrm{C} \ldots \ldots \ldots \quad 0,0120$

* Teneur en extrait sec déterminée à $100^{3}$ C. et sous un vide de $25-27$ pouces (inch) ( 85 à 125 millimètres).

La densité apparente du caséinate de calcium fut prise comme l'inverse du volume spécifique partiel. Pour calculer le volume spécifique partiel en partant des données pycnométriques, la concentration des solides en suspension, constitués en majeure partie par du caséinate de calcium, doit être déterminée correctement.

La quantité de lactalbumine et de globuline dans les laits écrémés est une constante; cette valeur est d'environ $0,55 \%$ (2). Le

(1) Pour la théorie concernant la détermination des courbes de distribution, au moyen de la centrifuge, voyez Svedberg et Rinde : Journ. Am. Chem. Soc., 46, 2681-2685 (1924); RINDE : "Tho Distribution of the Sizes of Particles in Gold Sols ", Diss. Upsala, 1928 ; et Svedrerg : "Colloid Chemistry ", Seconde Edition, pp. 178 et suiv. (1928).

(2) Rogers : "Fundamentals of Dairy Science », p. 43 (1928). 
sérum utilisé contenait $0,40 \%$ de protéines, d'où l'on conclut qu'une partie des protéines solubles est retenue par le filtre. S'il n'y avait pas eu de rétention de lactalbumine ni de globuline par le filtre, la teneur en protéines du sérum aurait été approximativement de $0,55 \%$ et la teneur en extrait sec approximativement de $6,37 \%$. Ainsi 3,03\% (9,40 - 6,37) représentent dans le lait écrémé la teneur de substances solides en suspension, en majeure partie constituées par du caséinate de calcium. En dehors ducaséinate de calcium, la fraction contient, sans doute, une petite quantité de matière grasse à un degré élevé de dispersion, quelques leucocytes et peut-être quelques phosphates colloïdaux.

D'autres données sur ces échantillons nous ont permis de calculer le poids du caséinate de calcium, qu'on a trouvé égal à $2,94 \%$. Partant de ces considérations et pour les déterminations en question, $3,03 \%$ a été choisi comme quantité totale de la phase en suspension. La valeur trouvée pour $V$, le volume spécifique partiel, était de 0,665 et son inverse, 1,504 , est la densité apparente du caséinate de calcium.

Les trois échantillons de lait écrémé : (3) témoin ; (2) échantillon chauffé pendant dix minutes à $65^{\circ} \mathrm{C}$. et (1) échantillon chauffé à $95^{\circ}$ C. pendant dix minutes), furent dilués au cinquième de la concentration primitive, avec du sérum de lait préparé par ultra-filtration, et centrifugés à une vitesse de 4.000 tours par minute. Des plaques photographiques Eastman Process furent utilisées pour enregistrer l'effet de la centrifugation. Comme

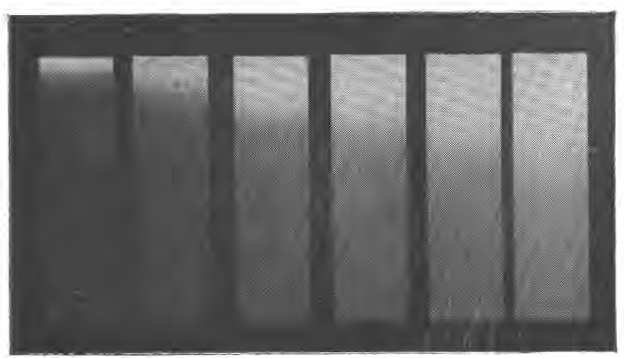

Fig. 1

S'édimentation de lait écrémé non chautfé. la source d'éclairage fut une lampe Pointolite, la radiation effective fut la région bleue du spectre. Des expositions d'une durée de dix à quinze secondes furent faites à des intervalles de dix minutes, chaque exposition fournissant des données sur lesquelles une distribution complète put être calculée. La figure 1 représente une série de photographies du progrès de la sédimentation du caséinate de calcium dans l'échantillon-témoin. La photographie à gauche représente la sédimentation durant la première période de dix minutes, et les photographies qui suivent représentent les résultats à des intervalles suivants de dix minutes, pendant une heure à partir du début. Les courbes de 
distribution du "poids optique " déterminées pour les trois échantillons sont représentées dans la figure 2. Les trois courbes de distribution sont suffisamment semblables pour être considérées comme presque identiques dans les limites de l'erreur expérimentale, quoiqu'il y ait une indication d'une légère modification de la moyenne du rayon vers une dimension plus petite de la particule, lorsque la température du chauffage préliminaire augmente - la dimension moyenne de $45 \mathrm{~m} \mu$ pour l'échantillon-témoin allant vers une dimen-

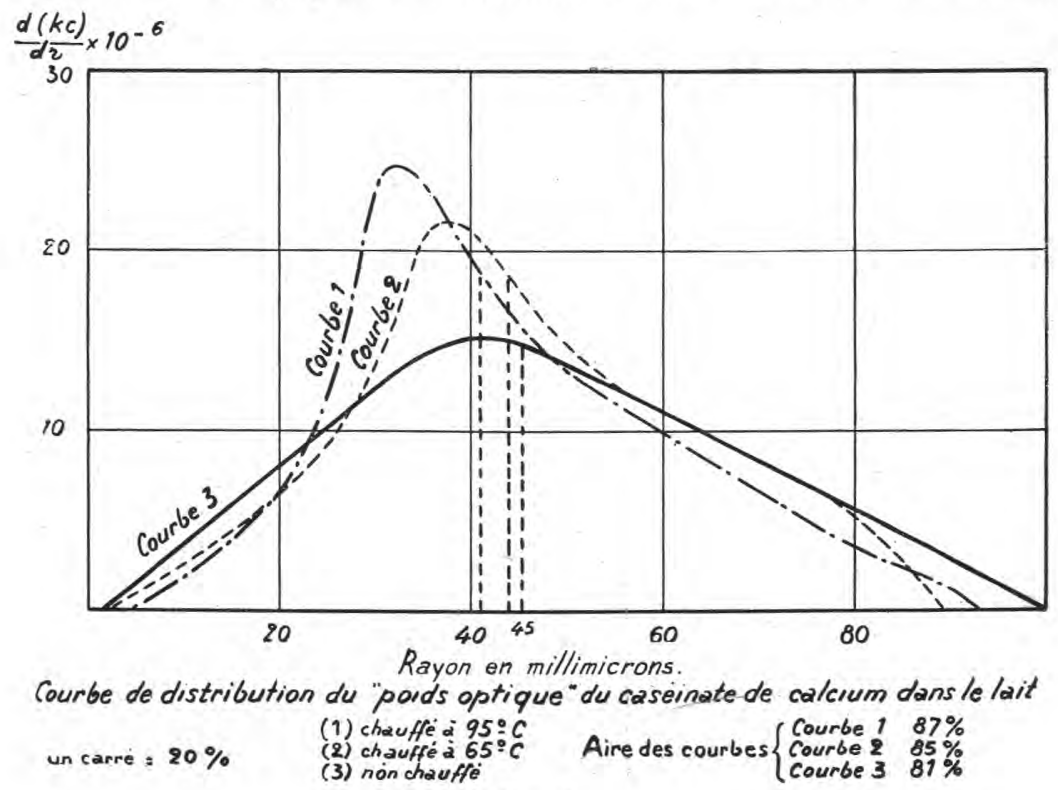

Fig. 2

sion moyenne de $41 \mathrm{~m} \mu$ pour l'échantillon chauffé à $95^{\circ} \mathrm{C}$. On pourrait difficilement affirmer que cette légère modification pût provoquer les notables différences de stabilité qui furent observées lorsque les échantillons furent soumis à l'épreuve de la coagulation après le chauffage préliminaire.

\section{DISCUSSION.}

Les résultats indiquent que les particules de caséinate de calcium dans le lait ont des dimensions variant d'environ $100 \mathrm{~m} \mu$ de rayon ( $200 \mathrm{~m} \mu$ de diamètre) jusqu'aux dimensions moléculaires, qui selon le travail récent de Svedberg, Carpenter, et Carpenter (1) sont de l'ordre de $4 \mathrm{~m} \mu$ de rayon pour la caséine. Le rayon moyen est de 40 à $50 \mathrm{~m} \mu$ (80 à $90 \mathrm{~m} \mu$ de diamètre). Cependant une quantité appréciable (environ $15 \%$, évaluée sur la base de l'absorption de

(1) Svedberg, Carpenter, et Carpenter : Journ. Am. Chem. Soc., 52, 241, 701 (1930). 
lumière) du matériel en suspension avait quitté le champ d'observation au cours des dix minutes écoulées entre la première et la seconde photographie. Cette fraction est probablement constituée par de petites quantités de matière grasse, des leucocytes, un peu de phosphate de calcium colloïdal et peut-être de grands agrégats de caséinate de calcium.

Dans un système polydispersé, dans lequel est présente la variété des dimensions du système caséinate de ealcium dont il est question ici, l'absorption de la lumière augmente rapidement avec l'accroissement du rayon, ceci en coneordance avee la dispersion de petites particules d'après RAYLEIGH. A cause de cela, les courbes de la figure 2 ne représentent pas le rapport vrai du poids du matériel au rayon, mais le rapport nommé " poids optique ", le terme "poids optique " indiquant une concentration apparente, qui est le produit de la constante d'absorption $k$ pour un rayon donné par la concentration $c$ du matériel ayant ee rayon. Si les courbes de distribution avaient présenté des différences notables, cette variation de l'absorption de lumière avec les dimensions des particules aurait rendu difficile la comparaison des différents échantillons. Comme en dépit de la grande variation de l'absorption de lumière selon les dimensions des particules les courbes sont pour ainsi dire identiques, nous sommes autorisés à conclure que la distribution des dimensions des particules du caséinate de calcium n'est que peu influencée par le chauffage préliminaire de courte durée jusqu'à une température de $95^{\circ} \mathrm{C}$.

Un chauffage prolongé du lait provoque la floculation ; on pourrait done raisonnablement supposer qu'il y a augmentation progressive de la dimension des particules avec l'augmentation de la durée et de la température du chauffage (1). Quoique ceci puisse se présenter dans les stades ultérieurs, les résultats mentionnés indiquent que les modifications des dimensions des particules ne sont pas d'importance première pour le phénomène de la stabilisation du lait, vis-à-vis de la chaleur, par un chauffage préliminaire; à moins qu'on admette que les légères modifications de la courbe de distribution dans le sens d'une dimension plus petite des particules soient susceptibles de produire les grandes modifications de la stabilité qui furent observées. Des modifications hydrolytiques pourraient alors être cause de la stabilisation.

\section{RÉSUMÉ}

$1^{0}$ Les courbes de distribution des dimensions des particules,

(1) Stedberg, Carpenter, et Carpenter, loc. cit., p. 708, ont observé une augmen. tation de la dimension de la molécule de caséine à $40^{\circ} \mathrm{C}$.; si une température si peu élevóe provoque une modification de la suspension du caséinate de caleium dans le lait écrémé, l'échantillon-témoin subit les mèmes modifications que les échantillons chauffés, parce que le lait a été chauffé à $40^{\circ} \mathrm{C}$. avant l'écrémage. 
en fonction du "poids optique ", ont été déterminées pour des échantillons de caséinate de calcium du lait écrémé dont le chauffage préliminaire avait été fait à des températures différentes.

$2^{\circ} \mathrm{La}$ majorité du matériel a un diamètre de moins de $200 \mathrm{~m} \mu$ avec une dimension moyenne d'environ $90 \mathrm{~m} \mu$.

$3^{\circ}$ Une petite quantité de matériel à particules plus grandes, probablement du phosphate de calcium colloïdal et de grands agrégats de caséinate de calcium, est également présente.

$4^{\circ}$ Le chauffage préliminaire jusqu'à $95^{\circ} \mathrm{C}$. a peu d'influence sur la courbe de distribution des dimensions des particules.

\title{
LES BESOINS EN LAIT DE L'ENFANT ET LES QUANTITÉS MINIMA NÉCESSAIRES A SA CROISSANCE (1)
}

\author{
par \\ M. L. RIBADEAU-DÚMAS \\ Médecin du Service maternel et infantile de la Salpètrière.
}

Les innombrables recherches concernant la valeur biologique des aliments ont confirmé l'observation séculaire qui avait mis en évidence la supériorité du lait sur toutes les formules alimentaires, quelles qu'elles soient.

Le lait représente un mélange harmonieux et complet des substances utiles à la croissance de l'organisme jeune. Les variantes de sa composition répondent seulement aux besoins des espèces. On a toujours pensé qu'au nourrisson humain convenait le lait de sa mère. Certaines circonstances économiques ou sociales conduisent à d'autres modes d'allaitement, généralement par le lait de vache. Celui-ci présente de grandes analogies avec le lait de femme, sans toutefois lui être tout à fait superposable : il nécessite done, pour être adapté aux facultés digestives du nourrisson, une correction qui est connue et universellement appliquée.

Mais, s'il est exceptionnel d'observer chez l'enfant nourri convenablement par sa mère, un trouble de croissance ayant quelque gravité, il n'en est pas de même pour l'enfant nourri au lait de vache. Il y a, en effet, entre le lait de vache et le lait de femme une différence que la clinique certifie, mais que l'analyse biologique n'a pas encore suffisamment précisée pour qu'on puisse, par une modification appropriée, tirer de l'allaitement artificiel les mêmes bienfaits que de l'allaitement naturel.

D'une manière générale, l'enfant normal qui est soumis à un allaitement convenablement dirigé, progresse régulièrement. Mieux

(1) Paru dans le Volume jubilaire du Prolesseur Ch. Porcher. 